J. Clin. Chem. Clin. Biochem.

Vol. 27, 1989, pp. 947-952

(C) 1989 Walter de Gruyter \& Co. Berlin - New York

\title{
Determination of Haemoglobin and Prothrombin Complex in Whole Blood using Optothermal Spectrometry
}

\author{
By P. Masson
}

Department of Clinical Chemistry, University Hospital, Lund, Sweden

H. Elwing, I. Lundström

Laboratory of Applied Physics, Linköping Institute of Technology, Linköping, Sweden

J. O. Karlsson

Department of Pharmacology, Linköping University Medical Hospital, Linköping, Sweden and

\section{P. Helander}

$A B$ Varilab, Huddinge, Sweden

(Received March 28/August 28, 1989)

Summary: Optothermal spectrometry measures the thermal energy produced as a result of absorption by molecules at a given modulation frequency and wavelength. Depending on the modulation frequency used, analysis can be performed in very thin layers (50-150 micrometers). A major advantage of optothermal spectrometry is that it is not very sensitive to light scattering. Haemoglobin in whole blood was measured without any reagent at $16 \mathrm{~Hz}$ and $2 \mathrm{~Hz}$ frequencies. The precisions (within-series, within-day and betweenday) were acceptable, and comparisons with reference methods were excellent. As opposed to the reference methods, optothermal spectrometric determinations were not affected by lipaemia. When measured continuously, the signal for haemoglobin increased due to the packing of erythrocytes towards the light source. By converting soluble fibrinogen into insoluble fibrin, the erythrocyte packing could either be inhibited or stopped, and this process could be monitored by assessing the change in the rate of signal increase for haemoglobin. This principle was utilized to analyse the prothrombin complex; the method was found to have acceptable precision and to be comparable to a routine method.

\section{Introduction}

The most commonly used optical methods for quantitative analyses are transmission and reflection spectrometry where the concentration of an analyte is measured either on the basis of its transmission or reflection properties. One drawback with these methods is that light-scattering in the sample strongly interferes with the measurement. This problem can be circumvented if the amount of light absorbed directly by the sample is measured. This can be achieved by detecting the temperature rise due to absorbed light and this principle forms the basis of optothermal spectrometry. Light impulses at a particular modulation frequency and a chosen wavelength are incident on a sample through a thin sapphire window $(1,2)$ as shown in figure 1. Each pulse causes a small temperature increase in the sample and this is transmitted to the sapphire. The subsequent thermal expansion of the sapphire is detected by a piezoelectric crystal and converted into an electrical signal. This signal has the same frequency as the modulation frequency of the light pulses and an amplitude depending upon 


\section{Wilhelm Friedrich}

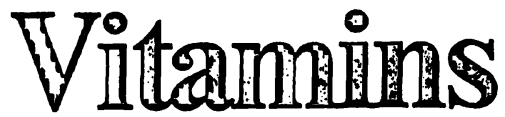

1988. $17 \mathrm{~cm}$ x $24 \mathrm{~cm}$. XII, 1062 pages. Hardcover. DM 380,-; approx. US \$225.00 ISBN 3110101447

A comprehensive, authoritative Handbook and reference source of all aspects of vitamins.

The book at a glance

- Complete and systematic overview of all water- and fat-soluble vitamins

- Introductory chapter presenting basic general information on the vitamins

- Numerous illustrations, formulas, tables, etc.

- Comprehensive and up-to-date bibliographic references at the end of each chapter

- Supplementary bibliography of publications that appeared subsequent to completion of the editorial work on the book

\section{From the Contents}

Introduction - Vitamin A and its Provitamins - Vitamin D - Vitamin E. Vitamin K Thiamin (Vitamin $\mathrm{B}_{1}$, Aneurin) · Vitamin $\mathrm{B}_{2}$ : Riboflavin and its Bioactive Variants Niacin: Nicotinic Acid, Nicotinamide, NAD (P) $\cdot$ Vitamin $B_{6} \cdot$ Folic Acid and Unconjugated Pteridines - Biotin · Pantothenic Acid - Vitamin $\mathbf{B}_{12} \cdot$ Vitamin $C$. Literature Supplement $\cdot$ Subject Index

\section{Potential audience}

Biochemists, Biologists, Clinicians, Pharmacologists, Toxicologists, Physiologists, Pharmacists, Nutritionists, Organic and Analytical Chemists. Institutes, Libraries 


\section{Concise Encyclopedia Biochemistry}

Second Edition, revised and expanded by Thomas Scott and Mary Eagleson

1988. $17 \mathrm{~cm} \mathrm{x} 24 \mathrm{~cm} .650$ pages. Hardcover. DM 148,-; approx. US $\$ 89.00$ ISBN 3110116251

The only single work of its kind in English, the Concise Encyclopedia of Biochemistry provides a comprehensive, yet compact, source of biochemical data and information for the researcher, teacher, and student.

Following a five-year program of collecting and editing new material, as well as the revision of existing entries, the author-editors and the publishers are pleased to announce the new expanded Second Edition of this valuable reference work.

Major entries concerning the latest developments in DNA structure, synthesis, sequencing, binding proteins and methods, oncogenes, lymphokines and other newly discovered regulatory peptides, structural proteins, inositol phosphates, and protein kinases have been added. Graphic illustration has been given high priority, so that regulatory processes, transport, subcellular structures, etc. are abundantly and clearly illustrated.

The coverage of plant biochemistry has also been greatly expanded. Another new addition is a section on buffers which will be useful to anyone involved in laboratory work. Because of its comprehensiveness and multidisciplinary nature, we are sure that you will find it an indispensable reference tool.

Special features of this edition include:

O Approximately 4,500 entries

O Up-to-date, comprehensive

O Coverage of medical, animal, microbial, plant, and physical biochemistry, natural products, molecular biology, molecular genetics, and biotechnology

- Hundreds of illustrations, including structural formulas, schemes, and metabolic pathways

- Over 100 tables

- Modern terminology based on standard sources, e.g., IUB Enzyme Nomenclature

- Standard biochemical abbreviations

- Extensive cross references with synonyms provided

- Literature references are cited to aid the reader in locating original sources

Potential audience: biochemists, clinical biochemists, clinical chemists, medical researchers, clinicians, plant scientists, experimental biologists, lecturers and students of the life sciences.

Price is subject to change without notice

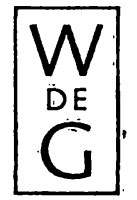

de Gruyter · Berlin • New Yorkz

Verlag Walter de Gruyter \& Co., Genthiner Str. 13, D-1000 Berlin 30, Tel.: (030) 260 05-0, Telex 184027

Walter de Gruyter, Inc., 200 Saw Mill River Road, Hawthorne, N. Y. 10532, Tel. (914) 747-0110, Telex 646677 


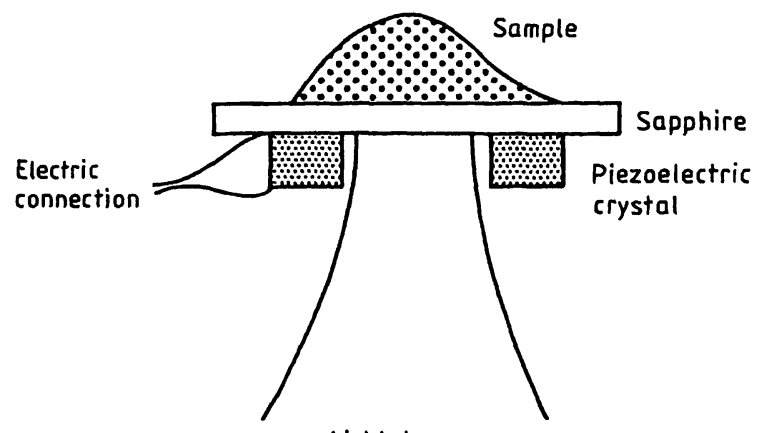

Light beam

Fig. 1. Cross sectional view of the optothermal detector.

the amount of light absorbed close to the sapphire (3). Light scattering changes the distribution of light in the sample and the amount of light close to the sapphire. However this effect is much less severe than that observed with other techniques, and can be compensated for by the calibration.

The thickness of the analysed layer depends on the speed at which the heat generated propagates from the sample to the sapphire. This distance is denoted as the thermal diffusion length (3) and depends inversely on the square root of the modulation frequency being used, a high frequency is required for analysis in a thin layer and vice versa. The present study was conducted either at 2 or $16 \mathrm{~Hz}$ frequency, where the thermal diffusion lengths in water are 149 and 52 micrometers, respectively. Hence optothermal spectrometry is well suited to analyse strongly absorbing samples such as whole blood, as well as samples where light scattering is a practical problem. The fact that only a small part of the sample is analysed (the sample is usually much thicker than the thermal diffusion length) gives an extra dimension for this technique. For whole blood, the number of erythrocytes within the analysis layer increases with time due to sedimentation, and this effect can be monitored (4). The sedimentation of erythrocytes has also been measured by a related technique called photoacoustic spectroscopy, where a microphone is used to measure pressure variations in a closed cell. Due to the instrumental design, the sedimentation is measured as a decreasing signal (5).

The use of optothermal spectrometry to analyse haemoglobin and the prothrombin complex using whole blood is presented here.

\section{(I) Determination of Haemoglobin}

\section{Materials and Methods}

Blood collected in EDTA for routine haematology was used. The optothermal spectrometer, constructed by $A B$ Varilab, operates at two frequencies $(16$ and $2 \mathrm{~Hz}$ ) by means of a mechan- ical chopper as shown in figure 2. A 20 Watt halogen lamp was used as the light source and a band pass filter (Schott, West Germany, filter BG $18,540 \pm 40 \mathrm{~nm}$ ) was used for the determination of haemoglobin. The sapphire window was built into a thermostated block for use at $37^{\circ} \mathrm{C}$. For analyses using $16 \mathrm{~Hz}$ frequency, $50 \mu \mathrm{l}$ of well mixed blood were applied to the sapphire window of the instrument and the lamp activated immediately. The instrument was calibrated using a patient pool of known haemoglobin concentration. To avoid a signal saturation effect at the lower frequency of $2^{\prime} \mathrm{Hz}$, well-mixed blood was diluted four-fold with deionized water containing $2 \mathrm{~g} / 1$ Sterox SE. The instrument was calibrated with diluted blood as mentioned above.

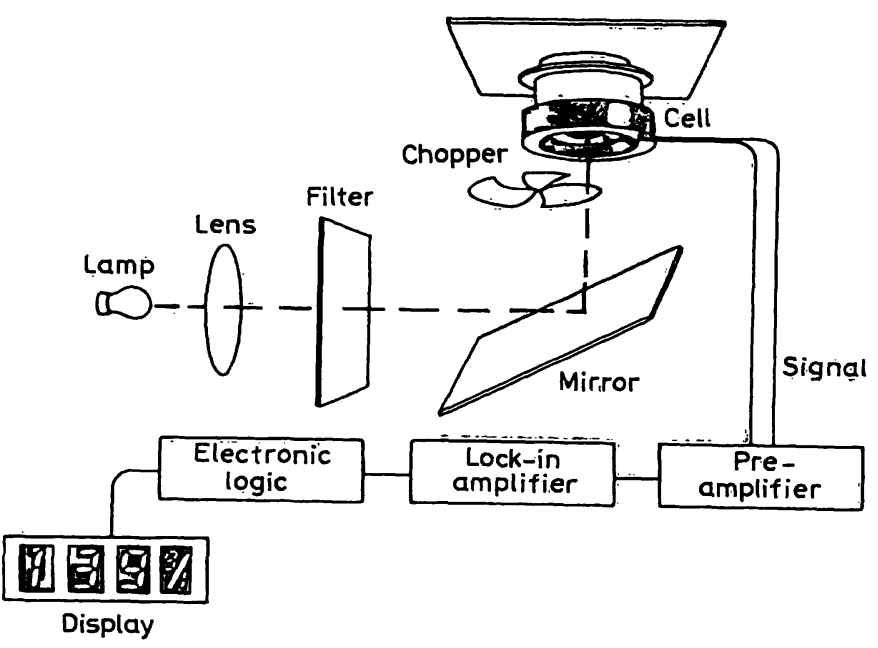

Fig. 2. Schematic representation of the optothermal spectrometer.

Within-series imprecision was calculated from consecutive analyses of three different samples. For within-day and betweenday imprecision, a stock standard was prepared by mixing several samples and diluting four fold with deionzed water containing $2 \mathrm{~g} / \mathrm{l}$ Sterox SE. Aliquots of $1 \mathrm{ml}$ were frozen at $-20^{\circ} \mathrm{C}$ and two aliquots were thawed daily for analyses five times in the morning and five times in the afternoon. Withinday imprecision was also tested using patient samples $(n=20)$ analysed during four days. Total imprecision was calculated from the available data.

To investigate linearity, three to four samples containing approximately the same amount of haemoglobin were mixed and centrifuged. The plasma was removed and replaced by a lesser volume of $150 \mathrm{mmol} / \mathrm{l} \mathrm{NaCl}$. Before the analysis at 2 and $16 \mathrm{~Hz}$ frequencies, the samples were diluted with $\mathrm{NaCl}$. Patient samples were analysed at these two frequencies and with the Coulter Counter model S-880 (Coulter Electronics, Luton, England), in which haemoglobin is analysed using the cyanide-methaemoglobin reaction (6). The haemoglobin concentrations were compared by least square regression analyses. The effect of lipaemia was investigated by adding different quantities of Intralipid (Kabi Vitrum, Stockholm, Sweden) to blood samples before analyses by optothermal spectrometry or with the Coulter S880.

\section{Results}

The coefficient of variation (CV) in \% is 100 times the quotient of the standard deviation (s) divided by the mean $(x)$. The ${ }_{n} C V$ s for within-series imprecision using optothermal spectrometry at 2 and $16 \mathrm{~Hz}$ frequencies varied between 0.2 and $3.3 \%$ (tab. 1). The coefficients of variation for within-day imprecision 
were $1.3 \%(16 \mathrm{~Hz})$ and $4.5 \%(2 \mathrm{~Hz})(\mathrm{tab} .2)$. Withinday CVs for patient samples were $3 \%$ at both frequencies. Between day CVs were approximately $5 \%$ (tab. 2). The coefficients of variation for total imprecision were $4.6 \%$ and $6.3 \%$, for 16 and $2 \mathrm{~Hz}$, respectively. The CVs for within-series, within-day, betweenday and total imprecision with the Coulter S-880, using a sample containing $120 \mathrm{~g} / \mathrm{l}$ haemoglobin, were $1.4,3.0,4.2$ and $3.7 \%$, respectively.

The signal response in optothermal spectrometry was linear up to $195 \mathrm{~g} / 1$ haemoglobin. Method comparisons between optothermal spectrometry and the Coulter Counter were excellent at both the frequencies tested (fig. 3). The comparison of the same patient samples $(\mathrm{n}=131)$ at $16 \mathrm{~Hz}(\mathrm{x})$ and $2 \mathrm{~Hz}(\mathrm{y})$ was satisfactory, giving the regression equation $y=1.02 x$ -2.1 (coefficient of correlation $r=0.981$ ).

The effect of lipaemia is shown in table 3 , where the results are presented as percentage recovery of haemoglobin in relation to the initial concentrations. Whereas analyses with the Coulter S-880 show a positive interference even at a relatively low degree of lipaemia, the results from optothermal spectrometry were not affected even in very lipaemic samples.

Tab. 1. Within series imprecision for haemoglobin

\begin{tabular}{lcccccc}
\hline & \multicolumn{3}{c}{$16 \mathrm{~Hz}$ frequency } & \multicolumn{3}{c}{$2 \mathrm{~Hz}$ frequency } \\
\hline $\mathrm{n}$ & 20 & 20 & 20 & 20 & 20 & 20 \\
$\overline{\mathrm{x}}, \mathrm{g} / 1$ & 24.9 & 123.4 & 160.1 & 32.3 & 111.8 & 154.7 \\
$\mathrm{~s}, \mathrm{~g} / 1$ & 0.13 & 2.22 & 2.56 & 1.10 & 0.22 & 0.46 \\
$\mathrm{CV}, \%$ & 0.5 & 1.8 & 1.6 & 3.3 & 0.2 & 0.3 \\
\hline
\end{tabular}

Tab. 2. Within-day and between-day imprecision for haemoglobin.

\begin{tabular}{|c|c|c|c|c|}
\hline & \multicolumn{2}{|c|}{$16 \mathrm{~Hz}$ frequency } & \multicolumn{2}{|c|}{$2 \mathrm{~Hz}$ frequency } \\
\hline & $\begin{array}{l}\text { Within= } \\
\text { day }\end{array}$ & $\begin{array}{l}\text { Between- } \\
\text { day }\end{array}$ & $\begin{array}{l}\text { Within- } \\
\text { day }\end{array}$ & $\begin{array}{l}\text { Between- } \\
\text { day }\end{array}$ \\
\hline$\dot{n}$ & 20 & 20 & 20 & 20 \\
\hline$\overline{\overline{\mathbf{x}}}, \mathrm{g} / \mathbf{1}$ & 33.6 & 34.0 & 33.9 & 34.5 \\
\hline $\mathrm{s}, \mathrm{g} / \mathrm{l}$ & 0.43 & 1.52 & 1.52 & 1.81 \\
\hline $\mathrm{CV}, \%$ & 1.3 & 4.5 & 4.5 & 5.3 \\
\hline
\end{tabular}

Tab. 3. Recoveries (\%) of haemoglobin in lipaemic samples.

\begin{tabular}{lllc}
\hline $\begin{array}{l}\text { Triacylglycerols } \\
(\mathrm{mmol} / \mathrm{l})\end{array}$ & $\begin{array}{l}\text { Coulter } \\
\text { S-880 }\end{array}$ & \multicolumn{2}{c}{ Optothermal spectrometry } \\
\cline { 3 - 4 } & & $\mathbf{1 6 \mathrm { Hz }}$ & $2 \mathrm{~Hz}$ \\
\hline 2.7 & 100 & 100 & 100 \\
5.0 & 108 & 100 & 99 \\
9.4 & 120 & 102 & 99 \\
16.0 & 138 & 103 & 100 \\
22.7 & 150 & 99 & 100 \\
\hline
\end{tabular}
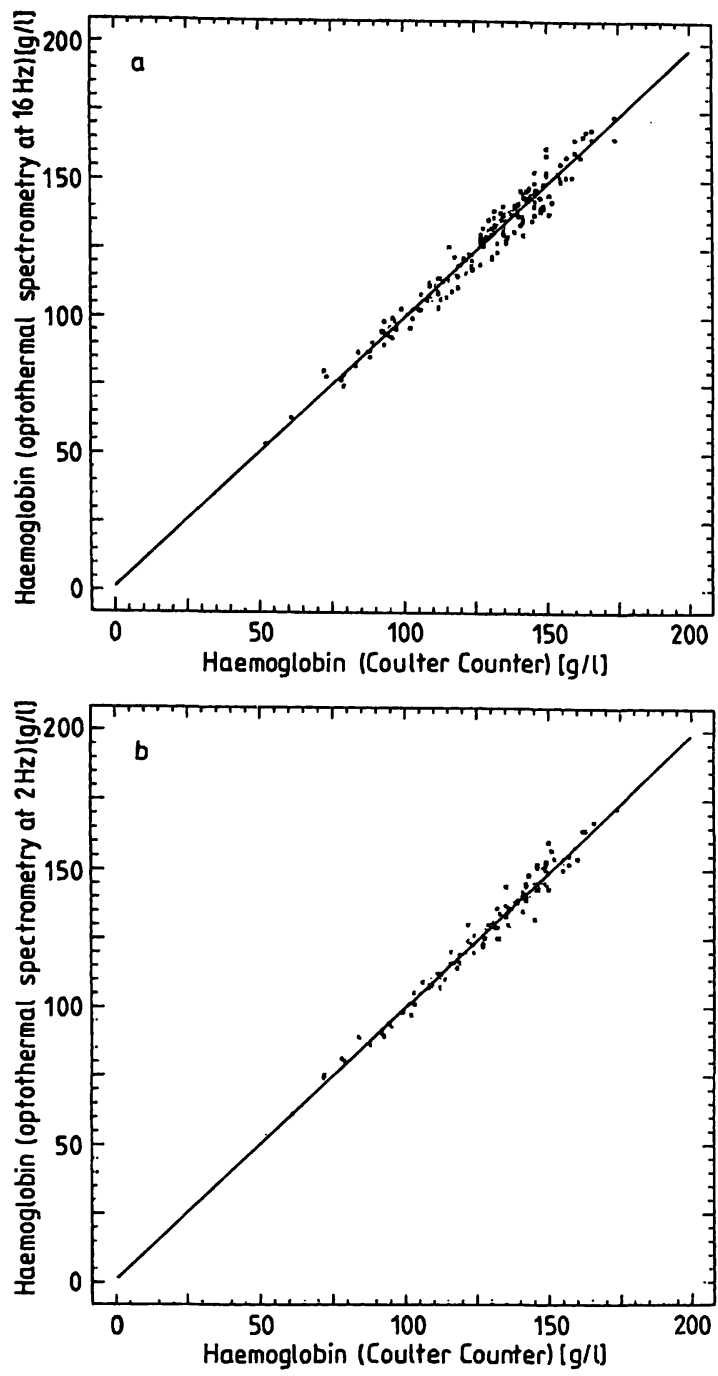

Fig. 3. Method comparisons:

a) Analyses of patient samples $(n=202)$ with the Coulter S-880 (x-axis) and by optothermal spectrometry at $16 \mathrm{~Hz}$ (y-axis). Least square regression analyses gave the equation $\mathrm{y}=0.98 \mathrm{x}+1.1(\mathrm{r}=0.983)$.

b) Analyses of patient samples $(n=131)$ with the Coulter S-880 ( $x$-axis) and by optothermal spectrometry at $2 \mathrm{~Hz}$ (y-axis). The regression equation was $y=0.99 x$ $+1.03(\mathrm{r}=0.990)$.

\section{(II) Determination of the Prothrombin complex}

\section{General}

The determination of the prothrombin complex is most commonly used as a "global" gauge of the functional activity of factors II, VII and X. Since these coagulation factors are vitamin $\mathrm{K}$-dependent, their concentrations are affected by the administration of Coumadin (Dicumarol, Warfarin) and thus the monitoring of the prothrombin complex has particular value in following anticoagulant therapy (7-9). The test is performed by adding tissue factor to recalcified plasma, whereby prothrombin is converted to thrombin and this converts soluble fibrinogen into insoluble fibrin. The formation of the fibrin clot can be determined either visually or with the help of some 
optical device. When haemoglobin is measured by optothermal spectrometry at $2 \mathrm{~Hz}$ frequency using a diluted sample, the light source is activated for a period of 20 seconds. If the sample was diluted with an isotonic solution and the light source activated continuously, the concentration of haemoglobin would increase gradually due to the sedimentation of the erythrocytes onto the sapphire window. When the same blood is mixed with a reagent used for the analysis of the prothrombin complex, the sedimentation of the erythrocytes either decreases or stops as soon as insoluble fibrin is produced, building a network and trapping the erythrocytes before they reach the sapphire window, thereby enabling quantitation of the coagulation time.

\section{Materials and Methods}

Blood samples collected in sodium citrate were analysed within four hours of sample collection. The contents of thrombotest reagent (batch No. 313, Nycomed \& Co, Oslo, Norway) were dissolved in $11 \mathrm{ml}$ of a solution containing $3.2 \mathrm{mmol} / 1 \mathrm{CaCl}_{2}$ and frozen at $-20^{\circ} \mathrm{C}$ as $100 \mu$ l aliquots. For the analysis, an aliquot was warmed to room temperature and $20 \mu \mathrm{l}$ of blood were added. A $40 \mu \mathrm{l}$ portion of the mixture was transferred immediately to the sapphire window of the optothermal spectrometer $\left(2 \mathrm{~Hz}\right.$ frequency, $37^{\circ} \mathrm{C}$ ) and the light source activated continuously. At the same time, the chart recorder coupled to the spectrometer was started (adjustment 2 volts, chart speed $1 \mathrm{~cm} / \mathrm{min}$ )

Within-series imprecision was tested at three different levels. Within-day and between-day imprecision were not tested since the coagulation factors are not stable to storage. To test linearity, a sample with a coagulation time of ca 50 seconds was diluted with $150 \mathrm{mmol} / \mathrm{l} \mathrm{NaCl}$ and analysed in triplicate with the instrument used for routine analyses (LODE LC-61, Holland) and optothermal spectrometry. For method comparisons, patient samples were analysed with the LODE LC-61 and by optothermal spectrometry, and the results were compared by least square regression analysis.

\section{Results}

The sedimentation of erythrocytes (analysed as haemoglobin) in a sample containing $20 \mu \mathrm{l}$ blood and $100 \mu \mathrm{l}$ of isotonic sodium chloride is shown in figure 4. When the sodium chloride is replaced by thrombotest reagent, the sedimentation rate is altered as soon as clot formation starts (fig. $4 \mathrm{~b}$ ). The point at which this rate of sedimentation alters is measured and represents the coagulation time in seconds.

The coefficients of variation for within-series analyses varied between 4.6 and $13.9 \%$ (tab. 4). The linearity extended over the range 48 to 220 seconds. This corresponds to coagulation activities of the prothrombin complex from $45 \%$ to less than $5 \%$. Results from the routine method and from optothermal spectrometry showed an acceptable level of agreement (fig. 5). The coagulation times were not affected by lipaemia in either method (tab. 5).

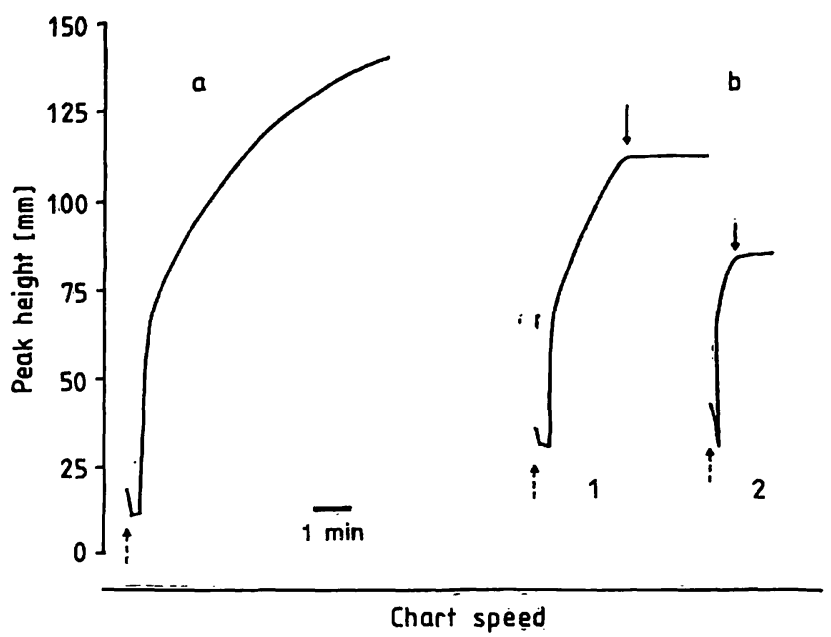

Fig. 4a. Sedimentation of erythrocytes.

Forty $\mu \mathrm{l}$ of blood diluted six-fold with $150 \mathrm{mmol} / \mathrm{l}$ $\mathrm{NaCl}$ were applied to the sapphire window of the optothermal spectrometer $\left(2 \mathrm{~Hz}, 37^{\circ} \mathrm{C}\right)$ and the lamp activated continuously. The haemoglobin in the erythrocytes was monitored on the chart recorder (speed: $1 \mathrm{~cm} / \mathrm{min}$ ). The start is indicated by the broken arrow.

Fig. 4b. Sedimentation of erythrocytes in the presence of thrombotest reagent.

Twenty $\mu \mathrm{l}$ of blood were mixed with $100 \mu$ of thrombotest reagent and $40 \mu \mathrm{l}$ applied to the sapphire window. The rest of the procedure was as in figure $4 \mathrm{a}$. (1) Sample with a coagulation time of $140 \mathrm{~s}$, (2) sample with a coagulation time fo $50 \mathrm{~s}$. Broken arrows indicate the start, completed arrows indicate the point of coagulation.

Tab. 4. Within-series imprecision for determination of the prothrombin complex.

\begin{tabular}{llll}
\hline \multicolumn{4}{c}{ Coagulation time (s) } \\
\hline $\mathrm{n}$ & 10 & 10 & 10 \\
$\overline{\mathrm{x}}$, seconds & 51.8 & 96.2 & 167.6 \\
$\mathrm{~s}$, seconds & 2.39 & 2.70 & 23.26 \\
$\mathrm{CV}, \%$ & 4.6 & 2.8 & 13.9
\end{tabular}

51.8 seconds represents a coagulation activity of $43 \%$ and 167.6 seconds an activity of $6 \%$.

\section{Discussion}

One of the biggest advantages with optothermal spectrometry is that haemoglobin can be determined in blood without derivatization, thereby eliminating the need for any reagent. The method measures haemoglobin predominantly as oxyhaemoglobin but all derivatives having an absorbance within the wavelength region 500 to $580 \mathrm{~nm}$ would also be included in the determination. Identical haemoglobin results were obtained either at room temperature $\left(\mathrm{ca} 20^{\circ} \mathrm{C}\right.$ ) or $37^{\circ} \mathrm{C}$. The choice of modulation frequency for optothermal spectrometry predetermines the method for haemoglobin analyses. Whole blood can be used directly at 16. $\mathrm{Hz}$ frequency. However, it is vital that the sample is thoroughly mixed and homogeneous before appli-

:

J. Clin. Chem. Clin. Biochem. / Vol. 27, 1989 / No. 12 


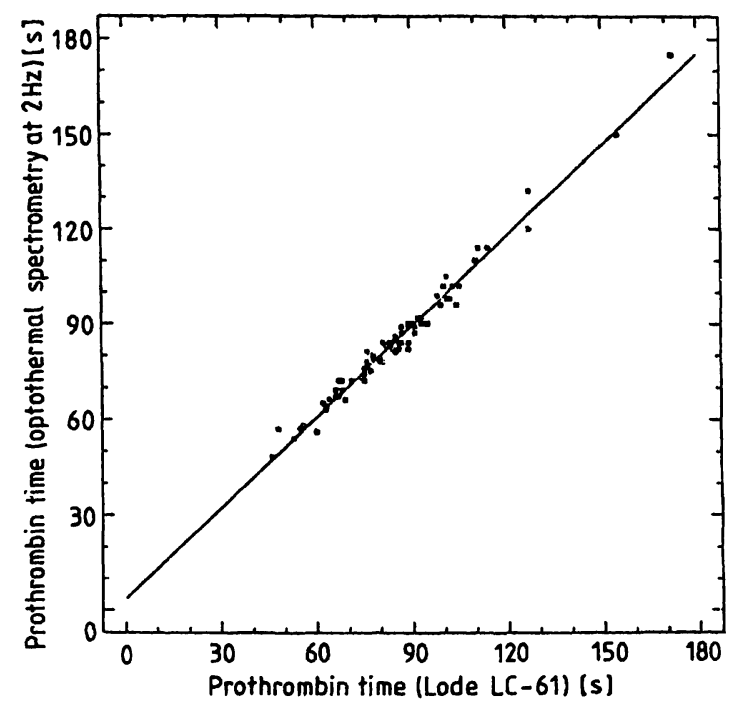

Fig. 5. Prothrombin time, method comparison.

Patient samples $(n=86)$ were analysed with LODE LC-61 ( $x$-axis) and optothermal spectrometry ( $y$-axis). Least square regression analyses gave the equation $y=0.96 x+3.46(r=0.989)$

Tab. 5. Effect of lipaemia.

\begin{tabular}{lll}
\hline $\begin{array}{l}\text { Triacylglycerols } \\
(\mathrm{mmol} / \mathrm{l})\end{array}$ & \multicolumn{2}{l}{ Coagulation time (s) } \\
\cline { 2 - 3 } & LODE & $\begin{array}{l}\text { Optothermal } \\
\text { Spectrometry }\end{array}$ \\
\hline 1.5 & 96 & 92 \\
3.8 & 95 & 91 \\
8.2 & 94 & 93 \\
14.8 & 95 & 93 \\
20.0 & 94 & 92 \\
\hline
\end{tabular}

cation to the sapphire window, and that the light source is activated immediately after sample application. Duplicate readings of the same blood sample should be avoided since the second value is always increased due to the packing of the erythrocytes on the sapphire window: Since the sensitivity of the instrument is increased three-fold at $2 \mathrm{~Hz}$ frequency, the blood sample needs to be diluted. Even though isotonic sodium chloride can be used for dilutions, the use of deionized water containing Sterox SE is preferred, since the sample is completely haemolysed, enabling duplicate readings without encountering the problem of erythrocyte packing. Optothermal spectrometry has been tested for sample volumes varying from 10 to $100 \mu l$ without loss of sensitivity and precision.

\section{References}

1. Helander, P. (1982) An open photoacoustic cell. J. Photoacoustics $1,103-120$.

2. McQueen, D. (1983) A simplified open photoacoustic cell. J. Phys. E., Sci. Instrum. 16, $738-739$.
Another advantage of optothermal spectrometry is its insensitivity to lipaemia. Since the measurement is performed in a thin layer of cells adjoining the sapphire window, it is feasible that lipids and chylomicrons, being low density particles, migrate towards the surface of the blood (away from the sapphire window) and thus have no effect on the measurements. Additionally, light scattering due to lipids which affects conventional photometry, has very little effect on optothermal spectrometry.

As shown in this study, both haemoglobin and the prothrombin complex were analysed with good precision, and the method comparisons were acceptable. A CV of $14 \%$ for prothrombin complex is not alarming since a difference in the coagulation time of 20 seconds in samples with long incubation times represents a difference in prothrombin complex of $1 \%$.

One possible drawback with optothermal spectrometry is that samples are applied directly on the sapphire window which needs wiping after every sample. We have therefore also evaluated disposable sample carriers. Essentially, these were paper blocks the size of normal photographic slides with a hole in the middle. A thin polymer film $(1.5 \mu \mathrm{m})$ was glued to one side producing a sample cell which could accommodate up to $200 \mu \mathrm{l}$ sample. The sample carriers could be placed with the cell directly over the sapphire window. The polymer film in contact with the window was pretreated with silicone to permit proper adhesion. However, the polymer film dampened the signals from the spectrometer by approximately 30 and $10 \%$ for 16 and $2 \mathrm{~Hz}$, respectively. This effect could be compensated mathematically for haemoglobin. No compensation was required for prothrombin complex, since this was measured as a change in the rate of sedimentation and variations in amplitude should have a minimal effect on the final outcome. Precision was acceptable at $2 \mathrm{~Hz}$ but poorer at $16 \mathrm{~Hz}$. Method comparisons against the routine methods were acceptable.

In conclusion, optothermal spectrometry represents a simple and practical method for the analysis of haemoglobin without derivatization, either in whole blood or in a haemolysed sample. Inhibition of sedimentation is used to measure the prothrombin complex.

3. Helander, P. (1986) Signal processing in optothermal spectroscopy. J. Appl. Phys. 59, 3339-3343.

4. Helander, P. \& Lundström, I. (1982) Whole blood - a sedimenting sample studied by photoacoustic spectrometry. J. Photoacoustics 1, 203-215. 
5. Poulet, P., Ouzafe, M. \& Chambron, J. (1988) Photoacoustic spectroscopy of human blood: Oxidation and sedimentation studies, In: Photoacoustics and photothermal phenomena. Proceedings of the 5th International Topical Meeting (Hess, $P$. \& Pelzl, J., eds.) pp. 538-541, Springer Verlag, Berlin.

6. van Kampen, E. J. \& Zijlstra, W. G. (1961) Standardization of haemoglobinometry II. The hemiglobincyanide method. Clin. Chim. Acta. 6, 538-544.
7. Owren, P. A. (1959) Thrombotest. A new method for controling anticoagulant therapy. Lancet $I I, 754-758$.

8. Peterson, C. E. \& Kwaan, H. C. (1968) Current concepts of warfarin therapy. Arch. Intern. Med. 146, 581- 584

9. Test insert from Nycomed \& Co Norway.

Dr P. Masșon

Department of Clinical Chemistry

University Hospital

S-221 85 Lund 